\title{
Role of Non Invasive Ventilation in Neurology Clinical Practice
}

\author{
Arun $\mathbf{R}^{1}$, J Nandana ${ }^{2}$ and Ashalatha Radhakrishnan ${ }^{2 *}$ \\ ${ }^{1}$ Sree Uthradom Thirunal Academy of Medical Sciences (SUTAMS), \\ Thiruvananthapuram, Kerala, India \\ ${ }^{2}$ Sree Chitra Tirunal Institute for Medical Sciences and Technology (SCTIMST), \\ Thiruvananthapuram, Kerala, India \\ *Corresponding Author: Ashalatha Radhakrishnan, Sree Chitra Tirunal Institute \\ for Medical Sciences and Technology (SCTIMST), Thiruvananthapuram, Kerala, \\ India. E-mail: drashalatha@sctimst.ac.in, ashalatharadhakrishnan@gmail.com
}

Received: January 11, 2021

Published: February 16, 2021

(C) All rights are reserved by Ashalatha

Radhakrishnan., et al.

\begin{abstract}
Non invasive ventilation (NIV) has become the standard practice for patients with neuromuscular respiratory failure. As mechanical ventilator can increase the risk of developing pneumonia or may cause lung injury, non invasive ventilation may be a good alternative for the same in certain neurological disorders. There are two main modalities of NIV: continuous positive airway pressure (CPAP) and Bi-level Positive Airway Pressure (BiPAP). Long-term non-invasive ventilation was introduced in the 1980s, initially for patients with poliomyelitis, muscular dystrophy or chest wall deformities like scoliosis. Bit in the last decade, sleep related disorders like the obesity-hypoventilation syndrome and sleep related disordered breathing of which sleep apneas has become one of the commonest reason for referral to most centres providing home based NIV especially. The use of NIV has been demonstrated to improve one's quality of life and survival in patients with many advanced neurological disorders and sleep disorders to a great extent.
\end{abstract}

Keywords: Noninvasive Ventilation; Critical Care; Neuromuscular Disorders; Sleep Disorders

\section{Introduction}

Non invasive ventilation (NIV) is defined as mechanical ventilation without the use of endotracheal intubation and has been increasingly established within intensive care units (ICU) during the last few decades. NIV has been recognised as an efficient therapeutic option in patients with both acute as well as chronic respiratory insufficiency due to neurological disorders.

Several neurological disorders like stroke, traumatic brain or spinal cord injury, and other toxic, infectious, inflammatory, metabolic or degenerative diseases involving the central or peripheral nervous system can trigger hypoxic and/or hypercapnic respiratory failure either directly or through pulmonary complications including pneumonia, pulmonary edema and traumatic pneumo- thorax [1]. In these patients, the weakness of respiratory muscles, or concomitant pulmonary complications due to oropharyngeal dysfunction causing aspiration or inefficient cough may lead to respiratory emergencies [2]. In all these neurological disorders, respiratory involvement tends to increase the burden of the existing disease, associated co-morbidities and mortality.

\section{Types of non invasive ventilation (NIV)}

Positive airway pressure (PAP) device is air in a compressor that delivers air pressure to specific levels as per the needs and demands of a person. The device interface can be a face mask (whole face mask or nasal mask), a nasal cannula or nasal pillows. PAP titration is done to select the lowest airway pressure that would eliminate all respiratory events, including apnea, hypopnea or a combination. 
There are two types of NIV, non-invasive positive-pressure (NIPPV) and negative-pressure ventilation (NINPV). NIPPV is commonly used in neurology clinical practice, it encompasses the delivery of oxygen at either constant or variable pressures via a face mask, such as continuous positive airway pressure (CPAP) and Bi-level positive airway pressure (BiPAP) or auto-titrating positive airway pressure (APAP) and high frequency oscillatory ventilation (HFOV). HFOV resembles a CPAP device in the sense that it basically applies a continuous mean air way pressure. Fresh gas flow of 40 to $60 \mathrm{lit} / \mathrm{min}$ occurs. The operator determines the overall oscillation amplitude. This pressure amplitude can be regulated up to 70 to $90 \mathrm{~cm}$ of water. HFVO offers the unique option of ventilating with very low tidal volume at relatively high mean airway pressure while maintaining acceptable $\mathrm{CO} 2$ clearance. This constitutes an extreme lung protection approach because tidal volume is minimized and the lung can be recruited by adequately high distending pressure without the risk of hyperinflation.

\section{Mechanisms of PAP functioning}

- Increasing the pharyngeal trans mural pressure ('pneumatic splint effect')

- $\quad$ Reducing the pharyngeal wall thickness and airway edema.

- Increasing airway tone by mechano-receptor stimulation.

- Increasing end-expiratory lung capacity producing 'tracheal tug effect'.

\section{CPAP}

It provides constant fixed positive pressure throughout inspiration and expiration, causing the airways to remain open and reduce the work of breathing [3]. This results in a higher degree of inspired oxygen than other oxygen masks.

\section{BiPAP}

As the name suggests provides differing airway pressure depending on inspiration and expiration. The inspiratory positive airways pressure (iPAP) is higher than the expiratory positive airways pressure (ePAP) [4]. Thus ventilation is provided mainly by iPAP, whereas ePAP recruits collapsed or under ventilated alveoli for gas exchange and allows removal of the exhaled gas.

\section{When to start NIV?}

Although successful application of NIV will largely depend on the particular clinical setting in which it is applied, some general considerations are in vogue. The importance of explaining the procedure to the patient, paying close attention to skin integrity, and appropriate mask interface selection can play a large role in the success of the intervention. Taking into consideration the indications and contraindications for NIV use is also crucial.

At least 2 of the following factors should be present:

Respiratory rate > 25 breaths/min;

Moderate to severe acidosis: pH 7.25 to 7.30 ; PCO2 45 to $60 \mathrm{~mm}$

$\mathrm{Hg}$;

Moderate to severe dyspnea with use of respiratory muscles and paradoxical breathing pattern

Table 1: Indications for NIV.

\begin{tabular}{|l|c|}
\hline Acute conditions & Chronic conditions \\
\hline $\begin{array}{l}\text { Myasthenia gravis exacerbation; } \\
\text { respiratory failure; }\end{array}$ & $\begin{array}{c}\text { Amyotrophic lateral scle- } \\
\text { rosis; }\end{array}$ \\
$\begin{array}{l}\text { Stupor or coma from overdose of } \\
\text { any drug(s); }\end{array}$ & $\begin{array}{c}\text { Muscular dystrophy; } \\
\text { Diaphragmatic failure from } \\
\text { phrenic nerve lesion; }\end{array}$ \\
$\begin{array}{l}\text { Postictal period after generalized } \\
\text { tonic-clonic seizure(s) or status } \\
\text { epilepticus of any type }\end{array}$ & $\begin{array}{c}\text { Sleep disorders-Sleep disor- } \\
\text { dered breathing with apnea } \\
\text { and/or destauration }\end{array}$ \\
\hline
\end{tabular}

Table 2: Some neurological indications for NIV.

\section{Absolute contraindications}

Respiratory arrest

Cardiac arrest

Cardiovascular instability (hypotension, dysrhythmias, acute myocardial infarction)

Non-respiratory organ failure (hemodynamic instability, severe encephalopathy, severe gastrointestinal bleeding)

Tracheoesophageal fistula

Inability to protect airway or high risk of aspiration

Uncooperative patient

Facial or head trauma or surgery

Relative contraindications

Copious secretions

Fixed nasopharyngeal abnormalities

Extreme obesity

Table 3: Contraindications for NIV. 
When should NIV be changed over to invasive ventilation?

Mechanical ventilation and endotracheal intubation are associated with increased risk of infectious complications, thus making NIV the preferred method of ventilatory treatment. Yet, there are circumstances where decision has to made regarding changing over from non-invasive to invasive ventilation.

Respiratory arrest

Respiratory rate $>35$ breaths $/ \mathrm{min}$

Severe dyspnea with use of accessory muscles paradoxical respiration

Severe hypoxemia: $\mathrm{PaO} 2<40 \mathrm{~mm} \mathrm{Hg}$ or $\mathrm{PaO} 2 / \mathrm{FiO} 2<200$

Severe acidosis $(\mathrm{pH}<7.25)$ and hypercapnia $(\mathrm{PaCO} 2>60 \mathrm{~mm}$ $\mathrm{Hg}$ )

Hypersomnolence, impaired mental status

Cardiovascular complications (hypotension, shock, heart failure)

Failure of noninvasive positive pressure ventilation

Table 4: Indications for changing over from NIV to invasive ventilation

Role of NIV in sleep disorders

Over the last three decades, NIV has been used for patients with sleep related breathing disorders such as obstructive sleep apnea (OSA), the overlap syndrome (COPD and obstructive sleep apnoea), obesity-hypoventilation syndrome (OHS), and sleep disorders associated with congestive heart failure (Cheyne-Stokes respiration) $[5,6]$.

\section{Central sleep apnea or complex sleep apnea syndrome}

All the following are based on nocturnal polysomnography (PSG): Apnea-hypopnea index (AHI) $>5$, central events $>50 \%$ of total, central events $\geq 5$ per hour, excessive daytime sleepiness or disrupted sleep and significant improvement on NIV and FiO2

\section{Obstructive sleep apnea}

Apnea- hypopnea index (AHI)/respiratory disturbance index (RDI) $\geq 15$ (minimum 30 events) or apnea--hypopnea index/ inspiratory disturbance index $<14$ with symptoms or cardiovascular risks (excessive daytime sleepiness, impaired cognition, mood disorders, insomnia, hypertension, ischemic heart disease, history of stroke)

Hypoventilation syndrome with or without obesity (Pickwickian syndrome)

Awake PaCO2 $\geq 45 \mathrm{mmHg}$ and $\mathrm{PaCO} 2 \geq 7 \mathrm{mmHg}$ greater during sleep or upon awakening or 02 saturation $\leq 88 \%$ for $\geq 5 \min (\geq 2$ $\mathrm{h}$ of recording on nocturnal PSG) with an apnea-hypopnea index $<5$

Table 5: Indications for NIV in sleep disorders.
Role of NIV in neuromuscular disorders

NIV has been used in patients with progressive neuromuscular diseases or serious abnormalities of the thoracic cage, with recognized benefits, which include an improved survival rate and an improved quality of life. The benefits of NIV in this type of patients includes improvement of daytime levels of blood gas (including hypercapnia), a reduction in the oxygen cost of breathing, an increase in the ventilatory response to increased carbon dioxide, and improved lung compliance [7].

Neuromuscular disorders can be divided into slowly and rapidly progressive. The slowly progressive conditions include non-progressive (e.g. poliomyelitis) or slowly progressive (e.g. some myopathies and muscular dystrophy). Here, physiological changes such as weight gain, chest infection, additional chronic cardiorespiratory pathology or sleep-disordered breathing, can lead to ventilatory decompensation, in the absence of progression of the underlying disease, thus requiring early ventilatory support. Among the progressive disorders are the Duchene muscular dystrophy and motor neuron disorders where NIV has been shown to be beneficial.

\section{Duchenne muscular dystrophy (DMD)}

The mean survival period following development of diurnal hypercapnia in DMD is 9.7 months if ventilatory support is not provided [8]. It is recommended [9] to initiate NPPV in symptomatic DMD patients with a daytime PaCO2 of $>6.0 \mathrm{kPa}$. There is no evidence that NPPV is effective when started as prophylactic therapy before the development of diurnal hypercapnia [10].

\section{Amyotrophic lateral sclerosis}

Amyotrophic lateral sclerosis (ALS) differs from DMD in being an acquired disorder, which tends to be more rapidly progressive. Hence the psychological and physical adaptations are more difficult. Patients with ALS do not to present with respiratory symptoms until vital capacity is limited to $50 \%$ of predicted. NIV is indicated in patients with diurnal PaCO2 $>6 \mathrm{kPa}$ [9] and may be of value in patients with normocapnia but with severe dyspnea or orthopnea due to respiratory muscle weakness. NIV is unlikely to benefit patients without symptoms or those with profound bulbar weakness.

Other uses of NIV in neurology are in Myasthenia gravis exacerbation, Guillain Barre syndrome with respiratory compromise, stupor from over dose, postictal period after generalised tonic clonic seizures or recurrent seizures and in phrenic nerve palsy of various causes. In status epilepticus, if the sensorium of the patient is altered at presentation or any time which can cause aspiration since the swallowing and cough reflex are deranged and also soon after the first and second line drugs for status fails (such as short-acting 
benzodiazapines including lorazepam, diazepam or midazolam; phenytoin or fos-phenytoin, sodium valproate, levetiracetam or other new generation drugs like lacosamide), the third line drugs i.e. anesthetic agents (viz. thiopentone, pentobarbital, midazolam or propofol) comes into play and patient needs to be mechanically ventilated. As the patient recovers and is gradually weaned off the mechanical ventilator, and at one stage where no more mechanical ventilation is needed, NIV used continuously and then intermittently is a good option for them to reach at their normal breathing pattern in room air.

\section{Role of NIV during extubation/post extubation period}

Extubation of patients from mechanical ventilation (MV) reduces the risk of serious complications associated with long term MV. But premature extubation may be equally detrimental to patient outcomes with the need for reintubation associated with a mortality of up to $40 \%$ in some patient groups [11]. The use of NIV in recently extubated patients provides an alternative to MV and may improve outcomes and mortality. NIV has been used as rescue therapy as well as preventative measure in recently extubated patients, who are at high risk for reintubation, in order to reduce the need for a period of further MV.

\section{Weaning from mechanical ventilation}

Weaning and extubation in neurocritical patients poses unique challenge due to the underlying neurological dysfunction and, indeed the incidence of extubation failure and reintubation in such patients has been observed to be approximately 10\%-15\% [12]. NIV has been utilised in patients who are difficult to be weaned off from ongoing MV, with the aim of reducing the risks associated with prolonged tracheal intubation. It has been suggested that NPPV could be used as a tool to help wean patients deemed not suitable for extubation from MV, by providing respiratory support without the need for sedation, neuromuscular block, and tracheal intubation in place of MV.

\section{Conclusions}

NIV is a useful alternative therapy to mechanical ventilation in many different neurological conditions causing acute/chronic respiratory failure. It may also have roles in preventing the development of respiratory failure in recently extubated ICU patients and in the postoperative period and also as a palliative treatment for patients with progressive neurological conditions. Anaesthetists and Neurologists should be aware of such an alternative as it helps to reduce respiratory distress and improve gas exchange without major complications or significant side effects such as infections. Treatment goal should be defined at the time of initiation of NIV. Thus, despite the increasing interest in its use, NIV should not be considered as a replacement for mechanical ventilation and it should not delay intubation and mechanical ventilation in those patients who fail to respond to or deteriorate on NIV.

\section{Bibliography}

1. Rabinstein AA and Wijdicks EF. "Warning signs of imminent respiratory failure in neurological patients". Seminar on Neurology 23.1 (2003): 97-104.

2. Boentert M., et al. "Respiratory involvement in neuromuscular disorders". Current Opinion on Neurology 30.5 (2017): 529537.

3. Nava S and Hill N. "Non-invasive ventilation in acute respiratory failure". Lancet 374.9685 (2007): 250-259.

4. Hörmann C., et al. "Biphasic positive airway pressure (BIPAP)a new mode of ventilatory support". European Journal of Anaesthesiology 11.1 (1994): 37-42.

5. Robert D and Argaud L. "Non-invasive positive ventilation in the treatment of sleep-related breathing disorders". Sleep Medicine 8.4 (2007): 441-452.

6. Theerakittikul T., et al. "Noninvasive positive pressure ventilation for stable outpatients: CPAP and beyond". Cleveland Clinic Journal of Medicine 77.10 (2010): 705-714.

7. Nickol AH., et al. "Mechanisms of improvement of respiratory failure in patients with restrictive thoracic disease treated with non-invasive ventilation". Thorax 60.9 (2005): 754-760.

8. Vianello A., et al. "Long-term nasal intermittent positive pressure ventilation in advanced Duchenne's muscular dystrophy”. Chest 105.2 (1994): 445-448.

9. “Clinical indications for non invasive positive pressure ventilation in chronic respiratory failure due to restrictive lung disease, COPD, and nocturnal hypoventilation--a consensus conference report". Chest 116.2 (1999): 521-534.

10. Raphael JC., et al. "Randomised trial of preventive nasal ventilation in Duchenne muscular dystrophy". Lancet 343.8913 (1994): 1600-1604.

11. Kulkarni AP., et al. "Extubation failure in intensive care unit: predictors and management". Indian Journal of Critical Care Medicine 12.1 (2008): 1-9. 
12. Rishi MA., et al. "Association of Extubation Failure and Functional Outcomes in Patients with Acute Neurologic Illness". Neurocritical Care 24.2 (2016): 217-225.

\section{Assets from publication with us}

- Prompt Acknowledgement after receiving the article

- Thorough Double blinded peer review

- Rapid Publication

- Issue of Publication Certificate

- High visibility of your Published work

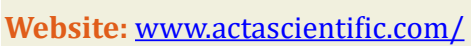

Submit Article: www.actascientific.com/submission.php

Email us: editor@actascientific.com

Contact us: +919182824667 